\title{
Pelvic arteriovenous malformation presenting as bloating and abdominal distension: a case report and literature review
}

\author{
Takayoshi Iijima ${ }^{1}$, Aya Tokinaga-Uchiyama ${ }^{1}$, Natsumi Fuchimukai ${ }^{1}$, Fumi Yanagisawa ${ }^{1}$, \\ Yuko Sonan $^{1}$, Ayaka Nakashima ${ }^{1}$, Mayu Shimomukai ${ }^{1}$, Kazunori Mukaida ${ }^{1}$, Hironori \\ Murakami $^{1}$, Yuta Kume ${ }^{1}$, Yukihiro Bonkohara ${ }^{1}$, and Mika Okuda ${ }^{1}$ \\ ${ }^{1}$ National Hospital Organisation Yokohama Medical Center
}

March 20, 2021

\begin{abstract}
Pelvic arteriovenous malformations (pAVMs) are rare lesions that sometimes pose diagnostic challenges. We report on a 50year-old woman with a pAVM presenting with only abdominal distension and bloating. The diagnostic tool was color Doppler transvaginal ultrasonography. Surgical excision of the AVM was performed, and there was no subsequent recurrence.
\end{abstract}

Pelvic arteriovenous malformation presenting as bloating and abdominal distension: a case report and literature review

Takayoshi Iijima $^{1}$, Aya Tokinaga-Uchiyama ${ }^{1}$, Natsumi Fuchimukai ${ }^{1}$, Fumi Yanagisawa $^{1}$, Yuko Sonan $^{1}$, Ayaka Nakashima ${ }^{1}$, Mayu Shimomukai ${ }^{1}$, Kazunori Mukaida ${ }^{1}$, Hironori Murakami ${ }^{2}$, Yuta Kume ${ }^{2}$, Yukihiro Bonkohara $^{2}$, Mika Okuda ${ }^{1}$

1. Department of Obstetrics and Gynecology, National Hospital Organization Yokohama Medical Center

2. Department of Vascular Surgery, National Hospital Organization Yokohama Medical Center

\section{INTRODUCTION}

Arteriovenous malformation (AVM) is an abnormal structure in which arteries and veins are directly connected without interposition of a capillary bed, forming a vascular nidus. 1 Although AVMs can occur anywhere in the body, pelvic AVMs (pAVMs) are rare and may pose a diagnostic and therapeutic challenge. While uterine arteriovenous malformations (uAVMs), located in the uterus, usually present with bleeding, pAVMs occurring outside the uterus show non-specific symptoms and are, thus, difficult to diagnose. 2, 3

We report a case of pAVM presenting with abdominal distension and evaluated as a pelvic mass using color Doppler transvaginal ultrasound. Surgical resection of the AVM was performed by vascular surgeons. Post-surgery, the patient was symptom-free and had no subsequent recurrence.

2 CASE

A 51-year-old woman, gravida 3, para 2, presented to our hospital with progressive and generalized abdominal distention since one month. She had not yet reached menopause. Her obstetric history included artificial abortion by surgical curettage 21 years prior, normal vaginal delivery 14 years prior, and an emergency cesarean section due to placental abruption 12 years prior. No other significant complaints were reported, except abdominal bloating. Her symptoms were exacerbated after meals and exertion, and alleviated on sitting. At admission, her vital signs were unremarkable and physical examination revealed a soft and non-tender abdomen. The pulsating mass was palpable on the left side of the pelvis but non-tender. 
Transvaginal ultrasonography showed an anechoic mass measuring $6 \mathrm{~cm} \times 3 \mathrm{~cm}$ in diameter on the left side of the pelvis, and color Doppler showed a mosaic pattern in the mass, representing turbulent blood flow (Fig. $1-1,1-2)$.

A diagnosis of pAVM was made using contrast-enhanced computed tomography (CT) and angiography (Fig. 2-1 and 2-2,3). The inflow vessels were the left internal iliac and inferior gluteal arteries. An abnormal vein around the AVM and a dilated left ovarian vein were also seen in the 3D CT angiography (Fig 2-2).

As complete resection was feasible due to the size and location of the AVM and fertility preservation was not required, surgical resection was planned. Surgery was performed by both gynecologists and vascular surgeons.

Intraoperative findings included an AVM measuring approximately $6 \mathrm{~cm} \times 3 \mathrm{~cm}$ in the retroperitoneal cavity. The uterus and ovaries were normal in size, and no other mass/cystic lesions were observed in the abdominal cavity. Initially, the left internal iliac artery was ligated to reduce the blood flow to the AVM. Hysterectomy and adnexectomy were then performed to achieve an optimal view of the surgical field. As the ligation of the internal iliac artery did not completely interrupt the blood flow to the AVM, multiple inflowing blood vessels from the lower gluteal artery were also ligated. The AVM was then successfully resected.

Histopathology of AVM showed evidence of congenital pAVM. Grossly, the specimen showed dilated vein-like blood vessels and arteries. Microscopically, some regions of the vascular wall were thinned, and some sections of the intima layer of the vascular wall were thickened via hyperplasia of smooth muscle fibers. This indicates abnormal differentiation of the vascular structures.

The symptoms of abdominal distension were resolved within two days of the surgery, and the abnormal blood flow in AVM and abnormal vasculature disappeared, as evidenced by the contrast-enhanced CT performed on post-operative day 6 (Fig. 4). Following this, the patient was discharged on post-operative day 7. A follow-up ultrasound after six months showed no evidence of a recurrent lesion.

\section{DISCUSSION}

The following two important points are highlighted in this case study: Abdominal bloating may potentially be the only symptom of pAVM, and transvaginal ultrasound and color Doppler are valuable tools for the detection of pAVMs.

The symptoms of pAVMs are usually non-specific, and patients may be asymptomatic and accidentally diagnosed.4 When symptomatic, they may present with abdominal or pelvic pain, lower urinary tract symptoms, such as hematuria and dysuria. 5, 6, 7 Uterine arteriovenous malformations (uAVMs) often present with bleeding. 8 In addition, the presence of abnormal shunts may also cause secondary heart failure and pelvic congestion syndrome (PCS). 9, 10 PCS is a clinical syndrome presenting as chronic pelvic pain that lasts for more than 3-6 months. Imaging studies may show dilation and regurgitation of the ovarian veins and dilation of the pelvic plexus.9

In the present case study, we report abdominal bloating as the only presenting symptom for pAVM. As abdominal bloating was completely relieved after surgery, AVM was considered to be the cause. Since the AVM was present in the retroperitoneum and was not large enough to obstruct the intestinal tract, it is highly unlikely that bloating was the result of compression of the intestinal tract. The symptoms may have been caused by the secondary PCS. The imaging findings were typical of PCS. This suggests that pelvic venous overload, congestion, and changes in hemodynamics may have contributed to the symptoms.

We conducted a literature review to explore the clinical scenarios associated with pAVMs using the MEDLINE database. The search was done using the strategic keyword "pelvic AVM." The case reports that did not mention symptomatology and those that revealed uterine AVM, and those that were written in languages other than English were excluded from the review (Table 1). A total of 31 articles(28 case reports and 3 review articles) were retrieved based on the inclusion and exclusion criteria. There were 43 cases, including the case reviewed here. Some of the articles did not provide patient age and sex. Approximately one-third 
of the cases reported (14 out of 37) were of female patients. The most common presenting complaint was pelvic and abdominal pain $(46 \%, 20$ out of 43$)$. Seven patients $(16 \%)$ were asymptomatic and diagnosed accidentally. Heart failure symptoms were reported in six cases (14\%) and were considered to be due to secondary PCS. None of the cases presented with abdominal distension as the chief complaint exept the case reviewed here.

Among the diagnostic strategies available for pAVMs, color Doppler transvaginal ultrasonography is a convenient and useful technique. It is less invasive and suitable for the screening of pAVMs. However, for a definitive diagnosis, dynamic CT imaging and angiography are required. 11

In the present case, transvaginal ultrasound revealed a cystic lesion with a mosaic pattern, suggesting turbulent blood flow. The internal iliac veins were visualized via the arterial phase of dynamic CT, and the inflow and outflow vessels were identified using angiography. Thus, these modalities serve as a valuable tool for the diagnosis and follow-up of vascular lesions such as AVM.

Abdominal bloating is a common and non-specific symptom that can be a manifestation of wide variety of ailments. Gastrointestinal causes are usually considered when associated symptoms such as constipation and nausea are present. Gynecological causes should also be ruled out as the gastrointestinal tract may be mechanically compressed by ovarian tumors, uterine fibroids, etc., giving rise to such symptoms. 12, 13 Thus, even if abdominal symptoms are the only chief complaints, a detailed investigation including the pelvic cavity aids in ruling out rare diseases, as in our present case.

In uAVM cases, color Doppler ultrasound shows a colored mosaic pattern in the non-echoic region of the myometrium, representing turbulent blood flow. 14, 15 Similarly, an anechoic lesion in the pelvis, as in the present case, can be diagnosed as AVM with similar findings on color Doppler imaging.

Although the natural history of pAVM is not well established, it is known to progress slowly and become symptomatic over time. 16 A delay in diagnosis may make the management more challenging. Additionally, if the secondary PCS progresses over time, heart failure may supervene. One study observed a large pAVM associated with cardiac failure, which was difficult to treat and recurred many times despite repeated treatments. 17 Therefore, for the early detection and efficient treatment of the disease, even unrelated and non-specific symptoms must be thoroughly investigated.

\section{CONCLUSION}

We diagnosed a patient, who presented with abdominal bloating and distention as chief complaints, with pAVM. Although pAVM is a rare disease, it may sometimes be associated with common symptoms such as abdominal distension. Non-invasive imaging studies, including color Doppler ultrasound, may play a key role in diagnostic screening for pAVMs. As early detection and treatment are crucial for pAVM, recognizing all the possible symptoms and the use of non-invasive rapid screening will prevent a missed diagnosis. This case report recognizes a rare manifestation of a known disease and aims to broaden the outlook of clinicians and surgeons.

\section{REFERENCES}

1. Walter A Wohlgemuth, Rene Muller-Wille, Veronika I Teusch, Oliver Dudeck, Anne M Cahill, Ahmad I Alomari, Wibke Uller. The retrograde transvenous push-through method: a novel treatment of peripheral arteriovenous malformations with dominant venous outflow. Cardiovasc Intervent Radiol 2015;38:623-631.

2. Rabia Hammad, Sidrah Nausheen, Mumtaz Malik. A Case Series on Uterine Arteriovenous Malformations: A Life-Threatening Emergency in Young Women. Cureus 2020;12:e9410.

3. Young Soo Do, Young-Wook Kim, Kwang Bo Park, Dong-Ik Kim, Hong Suk Park, Sung Ki Cho, Sung Wook Shin, Yang Jin Park. Endovascular treatment combined with emboloscleorotherapy for pelvic arteriovenous malformations. J Vasc Surg 2012;55:465-471.

4. Ashley Parrott, Rachel Lombardo, Nicole Brown, Justin T Tretter, Laura Riley, Kathryn Nicole Weaver. 
Cantu syndrome: A longitudinal review of vascular findings in three individuals. Am J Med Genet A 2020;182:1243-1248.

5. Allan M Conway, Khalil Qato, Jennifer Drury, Robert J Rosen. Embolization techniques for high-flow arteriovenous malformations with a dominant outflow vein. J Vasc Surg Venous Lymphat Disord 2015;3:178183.

6. Matteo Ripepi, Gianfranco Varetto, Lorenzo Gibello, Maria Antonella Ruffino, Paolo Fonio, Pietro Rispoli. Successful percutaneous transgluteal embolization of a complex arteriovenous malformation feeding a hypogastric artery aneurysm.J Vasc Surg Cases Innov Tech 2018;4:45-49.

7. M Tanaka, K Iida, S Matsumoto, T Takeuchi, K Yamaguchi, Y Nishimura, T Tominaga. A case of pelvic arteriovenous malformation in a male. Int J Urol 1999;6:374-376.

8. P-A Barral, M Saeed-Kilani, F Tradi, A Dabadie, J Izaaryene, J Soussan, J-M Bartoli, V Vidal. Transcatheter arterial embolization with ethylene vinyl alcohol copolymer (Onyx) for the treatment of hemorrhage due to uterine arteriovenous malformations. Diagn Interv Imaging 2017;98:415-421.

9. Mateus Picada Correa, Larissa Bianchini, Jaber Nashat Saleh, Rafael Stevan Noel, Julio Cesar Bajerski. Pelvic Congestion Syndrome and Embolization of Pelvic Varicose Veins. Review J Vasc Bras 2019;18:e20190061.

10. Chiara Borghi, Lucio Dell'Atti. Pelvic congestion syndrome: the current state of the literature. Arch Gynecol Obstet 2016;293:291-301.

11. Kenjiro Suzuki, Nobuyuki Tanaka, Takashi Ebine, Tetsuo Momma. Pelvic congenital arteriovenous malformation diagnosed by transrectal ultrasonography: A case report. Can Urol Assoc J 2012;6:E61-63.

12. Goff BA, Mandel L, Muntz HG. Melancon CH. Ovarian carcinoma diagnosis. Cancer 2000;89:2068-2075.

13. Elizabeth A Stewart, Wanda K Nicholson, Linda Bradley, Bijan J Borah. The burden of uterine fibroids for African-American women: results of a national survey. J Womens Health (Larchmt) 2013;22:807-816.

14. Pynar Polat, Selami Suma, Mecit Kantarcy, Fatih Alper, Akyn Levent. Color Doppler US in the evaluation of uterine vascular abnormalities. Radiographics. 2002;22:47-53.

15. Agata Szpera-Goździewicz, Karolina Gruca-Stryjak, Grzegorz H Bręborowicz, Mariola Ropacka-Lesiak. Uterine arteriovenous malformation - diagnosis and management. Ginekol Pol 2018;89:276-279.

16. Gresham T Richter, Adva B Friedman. Hemangiomas and vascular malformations: current theory and management. Int J Pediatr 2012;2012:645-678.

17. Tumay Bekci, Serap Yucel, Eser Turgut, Aysegul Idil Soylu. Giant Congenital Pelvic AVM Causing Cardiac Failure, Diplegia, and Neurogenic Bladder. Pol J Radiol 2015;80:388-390.

18. L D Vos, E P Bom, D Vroegindeweij, A V Tielbeek. Congenital pelvic arteriovenous malformation: a rare cause of sciatica. Clin Neurol Neurosurg 1995;97:229-232.

19. M Tanaka, K Iida, S Matsumoto, T Takeuchi, K Yamaguchi, Y Nishimura, T Tominaga. A case of pelvic arteriovenous malformation in a male. Int J Urol 1999;6:374-376

20. Zareh Kassardjian, Thierry Lebret, François Mellot, Jean-Marie Hervé, Philippe Barré, Pierre-Marie Lugagne, Antoine Scherrer, Henry Botto. Major complex pelvic arteriovenous malformation in a patient with Down syndrome. Urol Int 2002;69:145-149.

21. Robert A McCready, John W Fehrenbacher, Janet L Divelbiss, Ann Bryant, Scott Savader. Surgical resection of a large recurrent pelvic arteriovenous malformation using deep hypothermic circulatory arrest. J Vasc Surg 2004;39:1348-1350. 
22. Richard A Ashley, David E Patterson, Thomas C Bower, Anthony W Stanson. Large congenital pelvic arteriovenous malformation and management options. Urology 2006;68:203.e11-13

23. Kuri Suzuki, Daisuke Nishimi, Hajime Morioka, Masaharu Takanami. Hematospermia associated with congenital arteriovenous malformation of internal iliac vessels. Int J Urol 2007;14:370-372.

24. Andrew J Richards, Andrew Hatrick, Christopher G Eden. Large pelvic arteriovenous malformation complicating laparoscopic radical prostatectomy. Urology 2008;72:1359-1361.

25. S K Verma, D Bergin, C F Gonsalves, D G Mitchell. MRI detection of a female pelvic arteriovenous fistula after hysterectomy: treatment with superselective coil embolization. Br J Radiol 2008;81:e221-224.

26. Simon William Dubrey, Rowan Hillson, Maher Dahdal. High output heart failure caused by extensive arteriovenous malformation: problems and pregnancy. BMJ Case Rep 2009;2009:bcr07.2008.0546.

27. Yoshiko Watanabe, Toru Iwahashi, Naozumi Saiki, Nobusato Koizumi, Toshiya Nishibe, Hitoshi Ogino. Conservative therapy for surgically untreatable extensive arteriovenous malformation from the lower extremity to the pelvis with secondary consumptive coagulopathy. Ann Vasc Dis 2011;4:348-352.

28. Masamichi Koganemaru, Toshi Abe, Ryoji Iwamoto, Masako Suenaga, Kei Matsuoka, Naofumi Hayabuchi. Pelvic arteriovenous malformation treated by superselective transcatheter venous and arterial embolization. Jpn J Radiol 2012;30:526-529.

29. Bartłomiej Kędzierski, Grzegorz Nowak, Małgorzata Kuśmierska, Przemysław Jaźwiec, Andrzej Szuba. Giant congenital malformation of the perirectal plexus in computed tomography imaging - case report. Pol J Radiol 2013;78:50-53.

30. Kenji Murakami, Takayuki Yamada, Reiko Kumano, Yasuo Nakajima. Pelvic arteriovenous malformation treated by transarterial glue embolisation combining proximal balloon occlusion and devascularisation of multiple feeding arteries. BMJ Case Rep 2014;2014:bcr2013203492.

31. Aysun Erbahceci Salik, Filiz Islim, Ahmet Akgul, Barbaros Erhan Cil. Concomitant transarterial and transvenous embolization of a pelvic arteriovenous malformation using a new liquid embolic agent, squid-12 and detachable coils. Case Rep Vasc Med 2014;2014:972870.

32. Munemasa Okada, Masatoshi Kato, Kosuke Uchida, Yoko Sufu, Shinichi Okuda, Masafumi Yano, Naofumi Matsunaga. Transcatheter and percutaneous procedures for huge pelvic arteriovenous malformations causing high-output heart failure. J Cardiol Cases 2015;12:162-165.

33. Eric A Addo, Justin Emtage, Kamal Massis, David J Hernandez. A Congenital High Flow Arteriovenous Malformation of the Bladder Presenting With Polypoid Cystitis and Ureteral Obstruction. Urol Case Rep 2015;3:181-184.

34. Naiem Nassiri, Jones Thomas, Saum Rahimi. Fibrodysplastic implications for transvenous embolization of a high-flow pelvic arteriovenous malformation in Osler-Weber-Rendu syndrome. J Vasc Surg Cases $2015 ; 1: 16-19$.

35. M Saeed Kilani, V Lepennec, P Petit, G Magalon, D Casanova, J-M Bartoli, V Vidal. 2017. Embolization of peripheral high-flow arteriovenous malformations with Onyx. Diagn Interv Imaging 98:217-226.

36. Naiem Nassiri, Dustin T Crystal, Catherine Hoyt, Randy Shafritz. Chronic refractory venous ulcer exacerbated by a congenital pelvic arteriovenous malformation successfully treated by transarterial Onyx embolization. J Vasc Surg Venous Lymphat Disord 2017;5:417-420.

37. Matteo Ripepi, Gianfranco Varetto, Lorenzo Gibello, Maria Antonella Ruffino, Paolo Fonio, Pietro Rispoli. Successful percutaneous transgluteal embolization of a complex arteriovenous malformation feeding a hypogastric artery aneurysm. J Vasc Surg Cases Innov Tech 2018;4:45-49. 
38. Endovascular therapy of arteriovenous malformation in a male patient with severe post-coital pelvic pain. Bartosz Zabicki, Marte Johanne V Holstad, Nattakarn Limphaibool, Robert Juszkat. Pol J Radiol 2019;84:e258-e261.

39. Aikaterina Assimacopoulos, Tej I Mehta, Catherine Brockmeier, Douglas Yim. 2019. Recurrent, Idiopathic Spontaneous Hemoperitoneum Requiring Multiple Laparoscopic Evacuations: A Rare Case of Bleeding Adnexal Arteriovenous Malformation. Cureus 2019;11:e5490.

40. Atsushi Ugajin, Hiroyuki Fujii, Akifumi Fujita, Hiroyasu Nakamura, Akira Fujisaki, Hideharu Sugimoto. Transvenous embolization for a huge pelvic arteriovenous malformation associated with prominent outflow veins. Radiol Case Rep 2020;15:285-291.

41. Mitsuhiro Kishino, Kenji Nishida, Koichiro Kimura, Marie Takahashi, Shuichiro Nakaminato, Hiroko Kume, Hidetoshi Uchiyama, Toshifumi Kudo, Shuichi Kawada, Kouichi Mori, Ukihide Tateishi. Paravesical space arteriovenous malformation as a specific subgroup of pelvic vascular anomaly: a case series and review of literature. Jpn J Radiol 2020;38:434-439.

42. Tianhao Xie, Li Pan, Ming Yang, Zhaoxi Xu, Tao Wu, He Huang, Ming Li, Lianting Ma. Analysis of spinal angiograms that missed diagnosis of spinal vascular diseases with venous hypertensive myelopathy: the non-technical factors. Eur Spine J 2020;29:2441-2448.

43. Satonori Tsuneta, Daisuke Abo, Noriko Oyama-Manabe, Chihoko Miyazaki, Yuki Yoshino, Ryo Morita, Takeshi Soyama, Kohsuke Kudo. Visualization of Quantitative Flow Reduction with 4D-flow Magnetic Resonance Imaging in a Patient with Pelvic Arteriovenous Malformation After Transcatheter Arterial Embolization. Cardiovasc Intervent Radiol 2020;43:1557-1560.

\section{AUTHOR CONTRIBUTION}

TI: drafted the manuscript contributed and performed the surgery. AU: performed the surgery and revised the manuscript. AN: revised the manuscript.NF, FY, MS, YS, MO: contributed to the clinical management of the patient. KM, HM, YK, YB: contributed to the clinical management of the patient and performed the surgery.

\section{ACKNOWLEDGMENT}

Published with written consent of the patient. We would like to thank Editage (www.editage.jp) for English language editing.

\section{CONFLICT OF INTEREST}

The authors have no conflicts of interest to declare. 

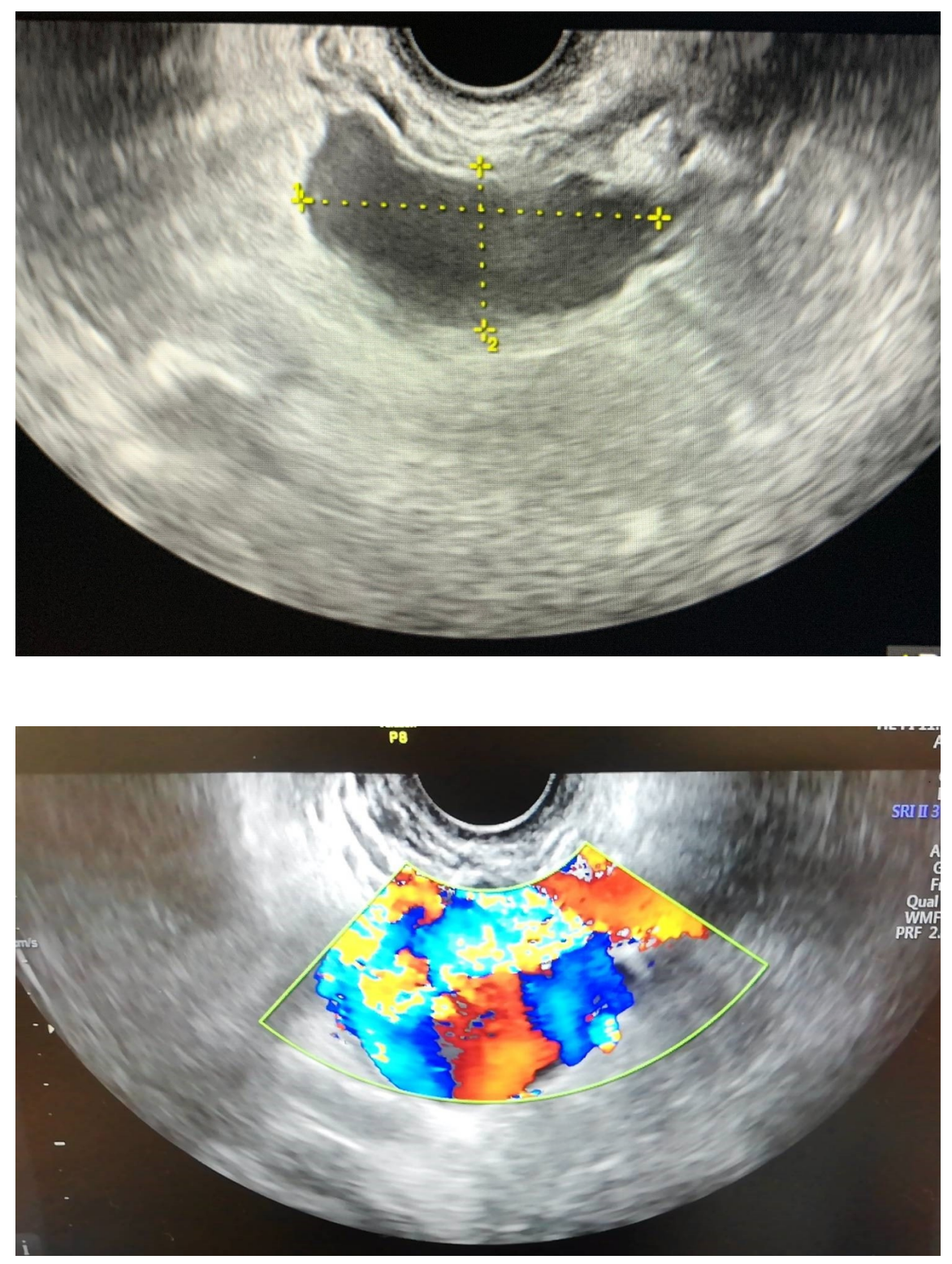

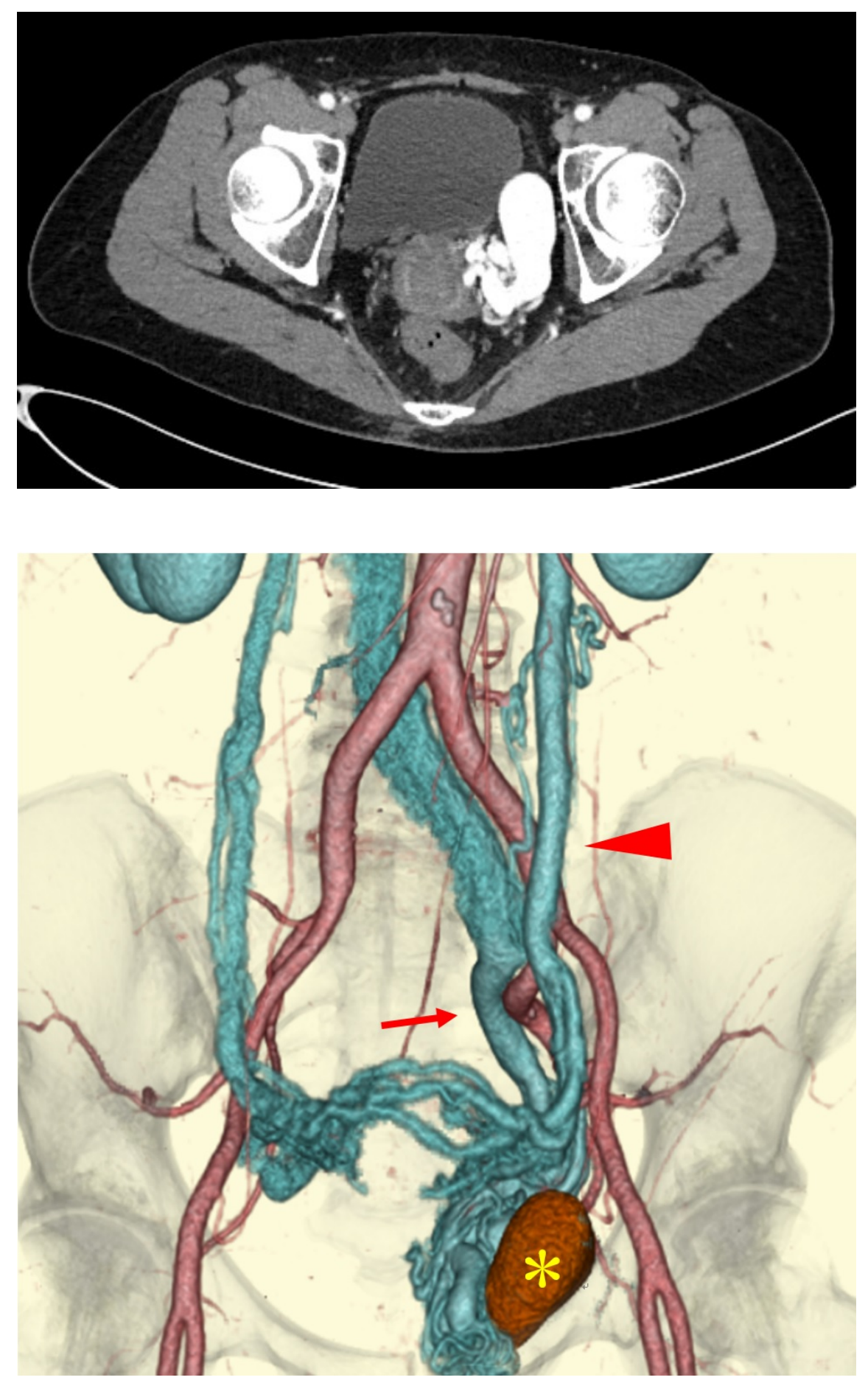


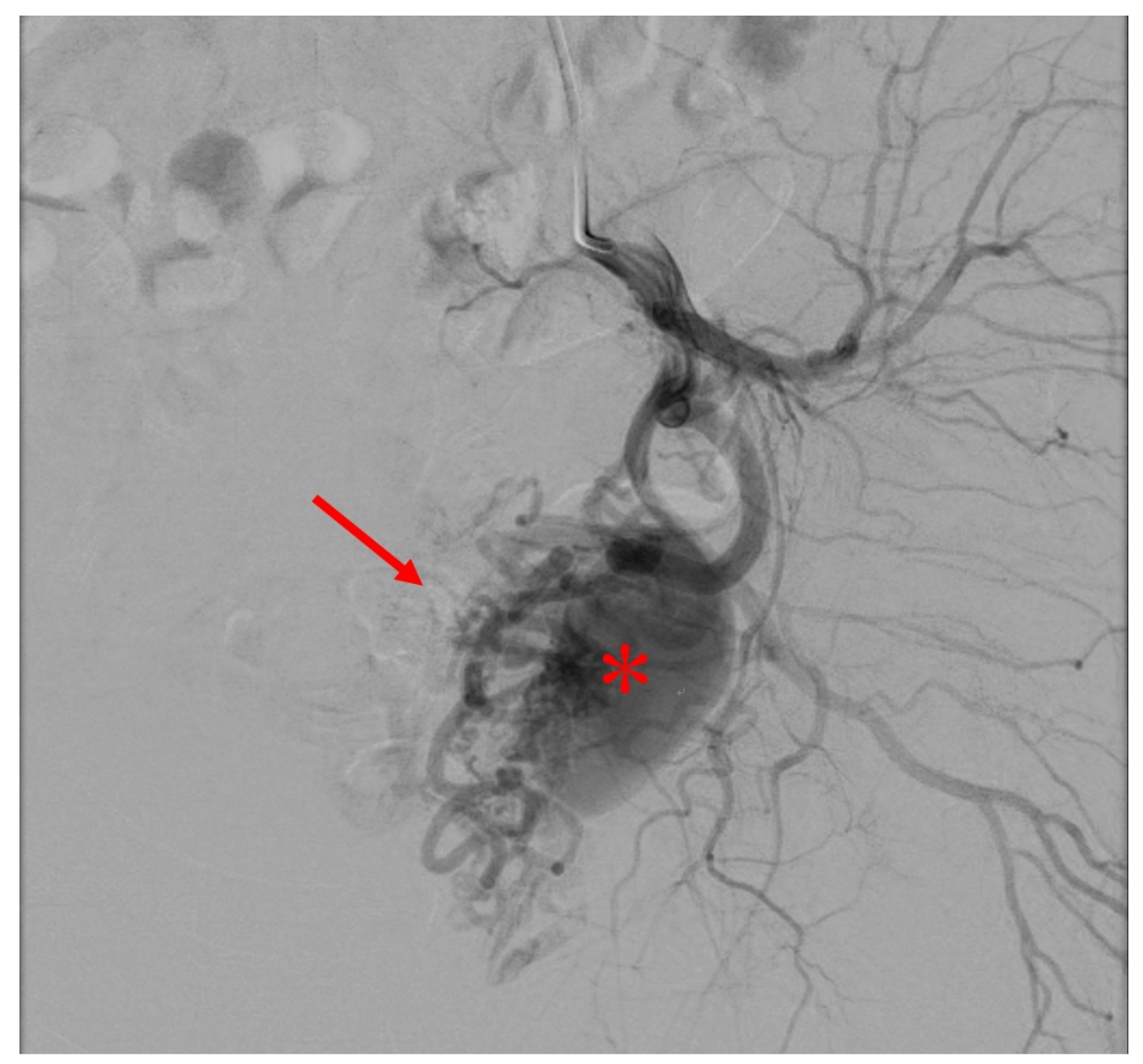




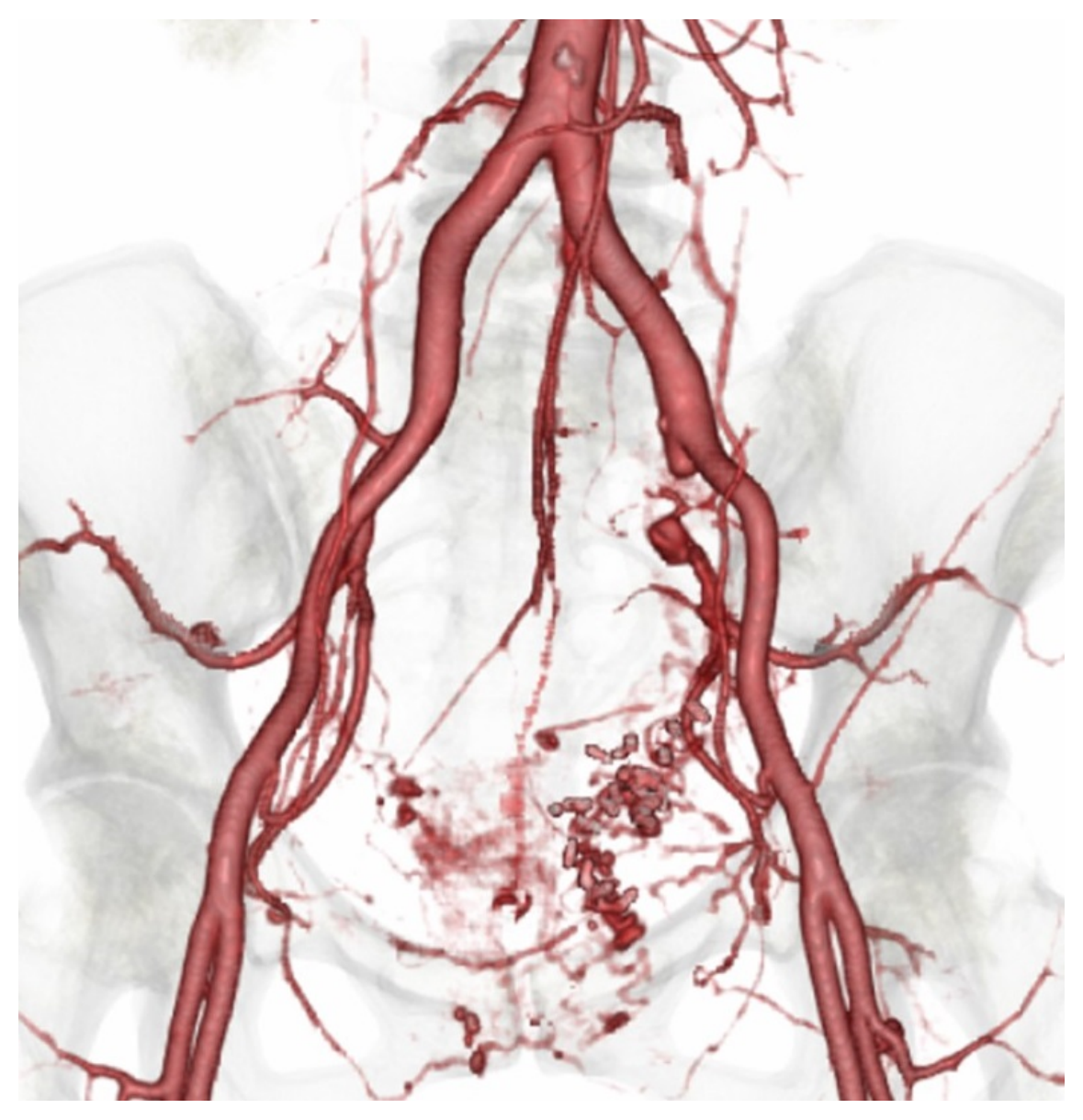

